\title{
Diversidad morfológica del sistema de conductos excretores de los peces teleósteos
}

\section{Morphological diversity of the excretory duct system of teleostean fishes}

\author{
Abraham Kobelkowsky \\ Laboratorio de Peces. Universidad Autónoma Metropolitana, Unidad Iztapalapa. Av. San Rafael Atlixco 186, Col. Vicentina, Iztapalapa. Apartado \\ postal 55-532. 09340 México, D.F. \\ Correspondencia: akd@xanum.uam.mx
}

Resumen. Mediante el análisis anatómico de 70 especies de teleósteos, pertenecientes a 62 géneros, 39 familias y 20 órdenes, se reconoce la diversidad morfológica de los conductos excretores y de la vejiga urinaria. Se define como un patrón morfológico general de los teleósteos la fusión de ambos conductos arquinéfricos en el conducto urinario común y, en los machos, la unión del conducto urinario común con el conducto espermático común, formándose el conducto urogenital. Se reconoce una relación inversa de la longitud de los conductos arquinéfricos y la longitud de los riñones. Se describe la diversidad de situaciones morfológicas del trayecto y fusiones de los conductos excretores. Se reconoce la diversidad de formas de vejiga urinaria. Resalta la "inversión" de la posición de los conductos urinarios y urogenitales en los Pleuronectiformes. Se sugiere el origen de la vejiga urinaria a partir del conducto urinario común. Se manifiesta en algunas especies el dimorfismo sexual en la forma de las papilas urinaria y urogenital.

Palabras clave: conductos urinarios, conductos urogenitales, vejiga urinaria, Teleostei.

\begin{abstract}
By means of an anatomical analysis from 70 species of teleostean fishes, belonging to 62 genera, and 39 families, and 20 orders, the morphological diversity of the urinary duct system is described. A general morphological teleostean pattern is defined as the fusion of both archinephric ducts in the common urinay duct, and the fusion in males of the common urinary duct with the common spermatic duct in the urogenital duct. A relation between the length of the kidney and of the archinephric ducts is recongnized. The trajectory of the urinary ducts is described, with the "reversal" of the orientation of the urinary and urogenital ducts in Pleuronectiformes being noteworthy. The origin of the urinary bladder from the common urinary duct is suggested.
\end{abstract}

Key words: Urinary ducts, urogenital ducts, urinary bladder, Teleostei.

\section{Introducción}

Los teleósteos constituyen el grupo más numeroso y diverso de todos los vertebrados, con cerca de 23637 especies, colocadas en 38 órdenes (Nelson, 1994). Los riñones en los teleósteos se localizan extraperitonealmente en el techo de la cavidad visceral y en posición ventral a la columna vertebral. Ambos riñones, derecho e izquierdo muestran diferentes grados de fusión entre sí, quedando, en general, la porción anterior separada en 2 lóbulos renales anteriores. La orina es drenada por los conductos arquinéfricos (o de Wolff) y almacenada temporalmente en una vejiga urinaria, de la cual parte un conducto urinario

Recibido: 13 abril 2006; aceptado: 28 septiembre 2006 común.

En los teleósteos, las gónadas en general están separadas de los riñones por la vejiga gaseosa (vejiga natatoria), encontrándose en los machos una relación entre el sistema excretor y el reproductor (Romer, 1962). Mientras que los ovarios son saculares y su túnica se prolonga para formar el gonoducto, los testículos son compactos y los recorren los conductos testiculares principales, que en su porción libre se les denomina conductos espermáticos.

Los trabajos sobre morfología del sistema urogenital de los teleósteos corresponden principalmente a especies holárticas (Rosen y Gordon, 1953; Gérard, 1954; Ogawa, 1961; Romer, 1962; Young, 1962; Peden, 1972; Lagler et al., 1977; Nelson, 1975; Chiasson, 1980; Nagahama, 1983; Moyle y Cech, 1988). Se han publicado diversas 
descripciones del sistema urogenital de especies de la República Mexicana por Castillo et al. (1988) en Bairdiella ronchus, Kobelkowsky (1999) en Bairdiella chrysoura y Stellifer lanceolatus, Kobelkowsky y Alva (2000) en Gambusia regani, Kobelkowsky (2000) en Achirus lineatus, Trinectes maculatus y Gymnachirus texae, Kobelkowsky (2004) en Bothus robinsi, Kobelkowsky (2005a) en Goodea atripinnis, y Kobelkowsky (2005b) en Astyanax fasciatus.

Considerando la elevada riqueza de especies de teleósteos de latitudes intertropicales, el presente trabajo tiene como objetivo mostrar parte de la diversidad morfológica de los conductos excretores de los teleósteos de México.

\section{Materiales y métodos}

Con el objeto de observar el sistema urogenital, se expuso la cavidad visceral de los ejemplares, retirando con instrumentos de disección, la piel, los músculos hipoaxiales, las costillas dorsales y las ventrales. Se observaron y retiraron el hígado, el tracto digestivo y la vejiga gaseosa. Las gónadas se localizaron entre la vejiga gaseosa y el intestino, y el riñón, en la parte ventral de la región precaudal de la columna vertebral.

El análisis anatómico macroscópico del sistema de conductos excretores se realizó en ejemplares correspondientes a 70 especies, 62 géneros, 39 familias y 20 órdenes de teleósteos: orden Elopiformes: Elops saurus (Elopidae); orden Anguilliformes: Ophichthus gomessi (Ophichthidae); orden Clupeiformes: Harengula jaguana, Brevoortia patronus, Dorosoma cepedianum (Clupeidae), Anchoa mitchilli, Cetengraulis edentulus (Engraulidae); orden Cypriniformes: Hybopsis boucardi, Carpiodes carpio (Cyprinidae); orden Characiformes: Astyanax fasciatus, Brycon guatemalensis, (Characidae); orden Siluriformes: Ictalurus balsanus, Ictalurus punctatus (Ictaluridae), Cathorops melanopus, Ariopsis felis, Bagre marinus (Ariidae), Rhamdia guatemalensis (Pimelodidae); orden Salmoniformes: Oncorhynchus mykiss (Salmonidae); orden Aulopiformes: Synodus foetens (Synodontidae); orden Batrachoidiformes: Opsanus beta, Porichthys porosissimus (Batrachoididae); orden Lophiiformes: Ogcocephalus radiatus (Ogcocephalidae); orden Mugiliformes: Mugil cephalus, Mugil curema (Mugilidae); orden Atheriniformes: Chirostoma humboltianum, Membras vagrans, Menidia beryllina (Atherinopsidae); orden Beloniformes: Strongylura marina, Strongylura notata, Strongylura timucu (Belonidae), Hyporhamphus roberti (Hemiramphidae); orden Cyprinodontiformes: Gambusia regani, Poeciliopsis infans, Belonesox belizanus (Poeciliidae), Goodea atripinnis (Goodeidae); orden Gasterosteiformes: Syngnathus scovelli (Syngnathidae); orden Synbranchiformes: Synbranchus marmoratus (Synbranchidae); orden Scorpaeniformes: Scorpaena plumieri (Scorpaenidae), Prionotus evolans, Prionotus maculatus (Triglidae); orden Perciformes: Centropomus undecimalis (Centropomidae), Micropterus salmoides (Centrarchidae), Lutjanus griseus (Lutjanidae), Eugerres plumieri, Eucinostomus argenteus, Diapterus auratus, Gerres cinereus (Gerreidae), Conodon nobilis (Haemulidae), Bairdiella chrysoura, Bairdiella ronchus, Stellifer lanceolatus, Cynoscion nothus, Menticirrhus saxatilis, Micropogonias undulatus (Sciaenidae), Petenia splendida (Cichlidae), Thalassoma lucasanum (Labridae), Gobionellus oceanicus (Gobiidae); orden Pleuronectiformes:Bothus robinsi(Bothidae), Citharichthys spilopterus, Syacium papillosum (Paralichthyidae), Achirus lineatus, Trinectes maculatus, Gymnachirus texae (Achiridae), Symphurus plagiusa, Symphurus civitatus (Cynoglossidae); orden Tetraodontiformes: Balistes capriscus (Balistidae), Diodon hystrix, Chilomycterus schoepfii (Diodontidae), Sphoeroides testudineus y Sphoeroides spengleri (Tetraodontidae).

El análisis microscópico de la conexión de las nefronas con los conductos colectores y de éstos con los conductos arquinéfricos se determinó de manera directa, bajo el microscopio estereoscópico, de la superficie ventral de los riñones de Oncorhynchus mykiss. La conexión de los conductos colectores con los arquinéfricos se observó mediante disección en Cathorops melanopus y Achirus lineatus.

Las ilustraciones se realizaron mediante una camera lucida acoplada a un microscopio estereoscópico Wild M3Z.

Los ejemplares utilizados en el presente trabajo corresponden a las colecciones de peces del Departamento de Biología de la Universidad Autónoma Metropolitana, Unidad Iztapalapa, del Laboratorio de Ictiología de la Escuela Nacional de Ciencias Biológicas del Instituto Politécnico Nacional y del Laboratorio de Bentos del Instituto de Ciencias del Mar y Limnología de la Universidad Nacional Autónoma de México.

\section{Resultados}

Se analizó la morfología del sistema de conductos excretores de 70 especies, correspondientes a 62 géneros, 39 familias y 20 órdenes de Teleostei.

En la superficie ventral del riñón de Oncorhynchus mykiss se observó sin practicar disección, la conexión de las nefronas con los conductos colectores y de éstos con 
los conductos arquinéfricos. Asimismo, la conexión de los conductos colectores con los arquinéfricos, se observó mediante disección en el riñón de Ariopsis felis y Achirus lineatus.

En términos generales, los conductos arquinéfricos de los teleósteos examinados se localizan cerca de los bordes del riñón y se desprenden de su extremo posterior, rodean a la vejiga gaseosa y descienden adosados al borde anterior del primer pterigióforo anal, hasta conectarse con la vejiga urinaria. Ambos conductos arquinéfricos se unen entre sí, constituyendo el conducto urinario común. De la vejiga urinaria parte o continúa, según el grupo, el conducto urinario común, el cual se abre al exterior mediante el orificio urinario en las hembras, mientras que en los machos se une al conducto espermático común, formando el conducto urogenital, que se abre al exterior mediante el orificio urogenital. En algunos grupos el orificio correspondiente se encuentra en el extremo de una papila.

En el presente trabajo se reconoció una diversidad morfológica de los conductos excretores de los teleósteos en los siguientes aspectos: 1) la longitud relativa de los conductos arquinéfricos, y la relación entre éstos y la forma de la cavidad visceral; 2) el grosor de los conductos arquinéfricos; 3) la trayectoria de los conductos arquinéfricos; 4) la fusión de ambos conductos arquinéfricos en el conducto urinario común; 5) la trayectoria del conducto urinario común; 6) la forma de la vejiga urinaria, y 7) el dimorfismo sexual de los conductos excretores.

1. Longitud relativa de los conductos arquinéfricos. a) se reconoce una relación inversa entre la longitud del riñón y la longitud de los conductos arquinéfricos. Los conductos arquinéfricos son relativamente largos en especies con los riñones compactos o cortos, localizados en la parte anterior de la cavidad visceral, como en Gambusia regani (Fig. 2 a). Los conductos arquinéfricos son cortos en especies con el riñón largo, como en Gobionellus oceanicus (Fig. 2 b) y Bairdiella chrysoura (Fig. 1), y b)se reconoce una relación entre la longitud de los conductos arquinéfricos y el "adelantamiento" de la aleta anal. En los machos de G. regani (Fig. 2 d) el adelantamiento de la aleta anal implica la reducción de la cavidad visceral y de los conductos arquinéfricos (Fig. 2 c). Comparando especies de la familia Atherinopsidae, se observa que los conductos arquinéfricos de Membras vagrans son más cortos que los de Menidia beryllina, debido al adelantamiento en ésta del esqueleto y musculatura anales, por lo que hacen un recorrido más largo (Fig. e, f).

2. Grosor de los conductos arquinéfricos. En todas las especies examinadas, los conductos arquinéfricos son relativamente delgados, sin embargo, en Synodus foetens son notablemente gruesos y con un aspecto interno similar al de su vejiga urinaria (Fig. 4 c, e).

3. Trayectoria de los conductos arquinéfricos. Después de abandonar el riñón, los conductos arquinéfricos se orientan hacia atrás y hacia abajo, siguiendo parte del contorno de la pared posterior de la cavidad visceral, hasta alcanzar la vejiga urinaria. Se reconocen 2 condiciones principales: a) los 2 conductos arquinéfricos rodean posteriormente la vejiga gaseosa y se ubican en el borde anterior de la primera espina hemal y el primer pterigióforo anal, como en Membras vagrans (Fig. 2 e), y b) los 2 conductos arquinéfricos rodean lateralmente a la vejiga gaseosa. En especies como Bairdiella chrysoura (Fig. 1) la rodean por ambos lados, mientras que en Onchorhynchus mykiss y Elops saurus, ambos conductos lo hacen por el mismo lado (Fig. 3 e).

4. Fusión de ambos conductos arquinéfricos en el conducto urinario común. De acuerdo con el cuadro 1, se reconocen 6 grados de unión entre los 2 conductos arquinéfricos: a) ambos salen de los riñones y alcanzan por separado la vejiga urinaria, después de la cual se forma el conducto urinario común, como en Diodon hystrix y Bairdiella ronchus (Fig. 3 a, b); b) ambos conductos salen por separado del riñón y se unen entre sí, justo antes de la vejiga urinaria, como en Goodea atripinnis (Fig. 3 c); c) ambos conductos se unen entre sí en algún punto entre el riñón y la vejiga urinaria, como en Ophichthus gomessi (Fig. 4 a). Los conductos colectores que en esta especie se unen en los arquinéfricos se encuentran en la parte externa del riñón; d) ambos conductos se unen entre sí en el punto de origen de la vejiga urinaria, como en Opsanus beta (Fig. 3 d) y Synodus foetens (Fig. 4 c); e) ambos conductos se unen entre sí en la parte ventral de la porción posterior del riñón, formando el conducto urinario común, como en Cathorops melanopus (Fig. 3 f); y f) ambos conductos se fusionan en el conducto urinario común, en la parte interna del riñón, como en Astyanax fasciatus (Fig. 3 g).

5. Trayectoria y longitud del conducto urinario común. En la mayoría de las especies el conducto urinario común se orienta hacia atrás y hacia abajo, como se ejemplifica con Bairdiella chrysoura (Fig. 5 b); sin embargo, algunas especies lo orientan hacia delante, como Ophichthus gomesii (Fig. 4 a), Achirus lineatus (Fig. 4 d), Citharichthys spilopterus y Bothus robinsi. Mientras que en la mayoría de las especies examinadas, el conducto urinario común es corto, este es largo en las especies en las que ambos conductos arquinéfricos se unen entre sí dentro o cerca del riñón como en Cathorops melanopus y Astyanax fasciatus (Fig. 3 f, g), Synodus foetens (Fig. 4 c) y Achirus lineatus (Fig. 4 d).

6. Forma de la vejiga urinaria. La vejiga urinaria de los teleósteos examinados se interpreta como una modificación del conducto urinario común, y su diversidad de formas se 
Cuadro 1. Diversidad morfológica de los conductos excretores y la vejiga urinaria en los teleósteos.1. Conductos arquinéfricos separados, desde el riñón hasta la vejiga urinaria.2. Conductos arquinéfricos fusionados entre sí, solamente en el contacto con la vejiga urinaria.3. Conductos arquinéfricos fusionados entre sí en algún punto entre el riñón y la vejiga urinaria. 4. Conductos arquinéfricos fusionados entre sí en el extremo del riñón.5. Conductos arquinéfricos fusionados entre sí en la superficie ventral del riñón. 6. Conductos arquinéfricos fusionados entre sí dentro del riñón. 7. Vejiga urinaria como un abultamiento simple o bilobular, que recibe los conductos arquinéfricos. 8. Vejiga urinaria sacular, que se desprende del punto en que se fusionan ambos conductos arquinéfricos en el conducto urinario común. 9. Vejiga urinaria como un ensanchamiento a lo largo del conducto urinario común. 10. Vejiga urinaria sacular, que se desprende del conducto urinario común.

\begin{tabular}{|c|c|c|c|c|c|c|c|c|c|c|}
\hline Especie & 1 & 2 & 3 & 4 & 5 & 6 & 7 & 8 & 9 & 10 \\
\hline Elops saurus (Elopidae) & & & & & $\mathrm{X}$ & & & & $\mathrm{X}$ & \\
\hline Ophichthus gomessi (Ophichthidae) & & & $\mathrm{X}$ & & & & & & & $\mathrm{X}$ \\
\hline Harengula jaguana (Clupeidae) & $\mathrm{X}$ & & & & & & & & $\mathrm{X}$ & \\
\hline Brevoortia patronus (Clupeidae) & $\mathrm{X}$ & & & & & & & & $\mathrm{X}$ & \\
\hline Dorosoma cepedianum (Clupeidae) & & & $\mathrm{X}$ & & & & & & $\mathrm{X}$ & \\
\hline Anchoa mitchilli (Engraulidae) & $\mathrm{X}$ & & & & & & & & $\mathrm{X}$ & \\
\hline Cetengraulis edentulus (Engraulidae) & & & & & & $\mathrm{X}$ & & & $\mathrm{X}$ & \\
\hline Hybopsis boucardi (Cyprinidae) & & & & $\mathrm{X}$ & & & & & $\mathrm{X}$ & \\
\hline Carpiodes carpio (Cyprinidae) & $\mathrm{X}$ & & & & & & & & $\mathrm{X}$ & \\
\hline Astyanax fasciatus (Characidae) & & & & & & $\mathrm{X}$ & & & & $\mathrm{X}$ \\
\hline Brycon guatemalensis (Characidae) & & & & & & $\mathrm{X}$ & & & & $\mathrm{X}$ \\
\hline Ictalurus balsanus (Ictaluridae) & & & & & $\mathrm{X}$ & & & & $\mathrm{X}$ & \\
\hline Ictalurus punctatus (Ictaluridae) & & & & & $\mathrm{X}$ & & & & $\mathrm{X}$ & \\
\hline Cathorops melanopus (Ariidae) & & & & & $\mathrm{X}$ & & & & $\mathrm{X}$ & \\
\hline Ariopsis felis (Ariidae) & & & & & $\mathrm{x}$ & & & & $\mathrm{X}$ & \\
\hline Bagre marinus (Ariidae) & & & & & $\mathrm{X}$ & & & & $\mathrm{X}$ & \\
\hline Rhamdia guatemalensis (Pimelodidae) & & $\mathrm{X}$ & & & & & & & & $X$ \\
\hline Oncorhynchus mykiss (Salmonidae) & & & & $\mathrm{X}$ & & & & & $\mathrm{X}$ & \\
\hline Synodus foetens (Synodontidae) & $\mathrm{X}$ & & & & & & & $\mathrm{X}$ & & \\
\hline Opsanus beta (Batrachoididae) & $\mathrm{X}$ & & & & & & & $\mathrm{X}$ & & \\
\hline Porichthys porosissimus (Batrachoididae) & $\mathrm{X}$ & & & & & & $\mathrm{X}$ & & & \\
\hline Ogcocephalus radiatus (Ogcocephalidae) & $\mathrm{X}$ & & & & & & $\mathrm{X}$ & & & \\
\hline Mugil cephalus (Mugilidae) & $\mathrm{X}$ & & & & & & $\mathrm{X}$ & & & \\
\hline Mugil curema (Mugilidae) & $\mathrm{X}$ & & & & & & $\mathrm{X}$ & & & \\
\hline Chirostoma humboldtianum (Atherinopsidae) & $\mathrm{X}$ & & & & & & $\mathrm{X}$ & & & \\
\hline Membras vagrans (Atherinopsidae) & $\mathrm{X}$ & & & & & & $\mathrm{X}$ & & & \\
\hline Menidia beryllina (Atherinopsidae) & $\mathrm{X}$ & & & & & & $\mathrm{X}$ & & & \\
\hline Strongylura marina (Belonidae) & $\mathrm{X}$ & & & & & & $\mathrm{X}$ & & & \\
\hline Strongylura notata (Belonidae) & $\mathrm{X}$ & & & & & & $\mathrm{X}$ & & & \\
\hline Strongylura timuси (Belonidae) & $\mathrm{X}$ & & & & & & $\mathrm{X}$ & & & \\
\hline Hyporhamphus roberti (Hemiramphidae) & $\mathrm{X}$ & & & & & & $\mathrm{X}$ & & & \\
\hline Gambusia regani (Poeciliidae) & $\mathrm{X}$ & & & & & & $\mathrm{X}$ & & & \\
\hline Poeciliopsis infans (Poeciliidae) & $\mathrm{X}$ & & & & & & $\mathrm{X}$ & & & \\
\hline Belonesox belizanus (Poeciliidae) & $\mathrm{X}$ & & & & & & $\mathrm{X}$ & & & \\
\hline Goodea atripinnis (Goodeidae) & & $\mathrm{X}$ & & & & & & & $\mathrm{X}$ & \\
\hline
\end{tabular}




\begin{tabular}{|c|c|c|c|c|c|}
\hline Syngnathus scovelli (Syngnathidae) & & $\mathrm{X}$ & & & $\mathrm{X}$ \\
\hline Synbranchus marmoratus (Synbranchidae) & $\mathrm{X}$ & & $\mathrm{X}$ & & \\
\hline Scorpaena plumieri (Scorpaenidae) & $\mathrm{X}$ & & & & \\
\hline Prionotus evolans (Triglidae) & $\mathrm{X}$ & & & & \\
\hline Prionotus maculatus (Triglidae) & $\mathrm{X}$ & & & & \\
\hline Centropomus undecimalis (Centropomidae) & $\mathrm{X}$ & & $\mathrm{X}$ & & \\
\hline Micropterus salmoides (Centrachidae) & $\mathrm{X}$ & & $\mathrm{X}$ & & \\
\hline Lutjanus griseus (Lutjanidae) & $\mathrm{X}$ & & $\mathrm{X}$ & & \\
\hline Eugerres plumieri (Gerreidae) & $\mathrm{X}$ & & $\mathrm{X}$ & & \\
\hline Eucinostomus argenteus (Gerreidae) & $\mathrm{X}$ & & $\mathrm{X}$ & & \\
\hline Diapterus auratus (Gerreidae) & $\mathrm{X}$ & & $\mathrm{X}$ & & \\
\hline Gerres cinereus (Gerreidae) & $\mathrm{X}$ & & $\mathrm{X}$ & & \\
\hline Conodon nobilis (Haemulidae) & $\mathrm{X}$ & & $\mathrm{X}$ & & \\
\hline Bairdiella chrysoura (Sciaenidae) & $\mathrm{X}$ & & $\mathrm{X}$ & & \\
\hline Bairdiella ronchus (Sciaenidae) & $\mathrm{X}$ & & $\mathrm{X}$ & & \\
\hline Stellifer lanceolatus (Sciaenidae) & $\mathrm{X}$ & & $\mathrm{X}$ & & \\
\hline Cynocion nothus (Sciaenidae) & $\mathrm{X}$ & & $\mathrm{X}$ & & \\
\hline Menticirrhus saxatilis (Sciaenidae) & $\mathrm{X}$ & & $\mathrm{X}$ & & \\
\hline Micropogonias undulatus (Sciaenidae) & $\mathrm{X}$ & & $\mathrm{X}$ & & \\
\hline Petenia splendida (Cichlidae) & $\mathrm{X}$ & & $\mathrm{X}$ & & \\
\hline Thalassoma lucasanum (Labridae) & $\mathrm{X}$ & & $\mathrm{X}$ & & \\
\hline Gobionellus oceanicus (Gobiidae) & $\mathrm{X}$ & & & & $\mathrm{x}$ \\
\hline Bothus robinsi (Bothidae) & & $\mathrm{X}$ & & $\mathrm{X}$ & \\
\hline Citharichthys spilopterus (Paralichthyidae) & & $\mathrm{X}$ & & $\mathrm{X}$ & \\
\hline Syacium papillosum (Paralichtyidae) & & $\mathrm{X}$ & & $\mathrm{X}$ & \\
\hline Achirus lineatus (Achiridae) & & $\mathrm{X}$ & & & $\mathrm{X}$ \\
\hline Trinectes maculates (Achiridae) & & $\mathrm{X}$ & & & $\mathrm{X}$ \\
\hline Gymnachirus texae (Achiridae) & & $\mathrm{X}$ & & & $x$ \\
\hline Symphurus plagiusa (Cynoglossidae) & $\mathrm{X}$ & & $\mathrm{X}$ & & \\
\hline Symphurus civitatus (Cynoglossidae) & $\mathrm{X}$ & & $\mathrm{X}$ & & \\
\hline Balistes capriscus (Balistidae) & $\mathrm{X}$ & & $\mathrm{X}$ & & \\
\hline Diodon hystrix (Diodontidae) & $\mathrm{X}$ & & $\mathrm{X}$ & & \\
\hline Chilomycterus schoepfii (Diodontidae) & $\mathrm{X}$ & & $\mathrm{X}$ & & \\
\hline Sphoeroides testudineus (Tetraodontidae) & $\mathrm{X}$ & & $\mathrm{X}$ & & \\
\hline Sphoeroides spengleri (Tetraodontidae) & $\mathrm{X}$ & & $\mathrm{X}$ & & \\
\hline
\end{tabular}

puede agrupar en 7 categorías: a) ensanchamiento de un tramo del conducto urinario común, como en Cathorops melanopus (Fig. 3 f), Citharichthys spilopterus y Bothus robinsi; b) formación sacular media e impar que recibe al conducto urinario común en Goodea atripinnis (Fig. 3 c) o a los 2 conductos arquinéfricos en Gambusia regani (Fig. 5 c); c) formación sacular lateral impar, alargada y orientada hacia delante, como en Ictalurus punctatus,
Astyanax fasciatus (Fig. 3 g), Opsanus beta (Fig. 3 d), Syngnathus scovelli (Fig. 4 b) y Elops saurus (Fig. 3 e); d) formación tubular media y notablemente larga, como en Synodus foetens (Fig. 4 c). En esta especie los conductos arquinéfricos tienen un aspecto interno similar al de la vejiga urinaria (Fig. 4 e); e) estructura bilobular como en Bairdiella ronchus (Fig. 3 b), y f) formación sacular que se orienta hacia atrás de manera extravisceral, siendo impar 


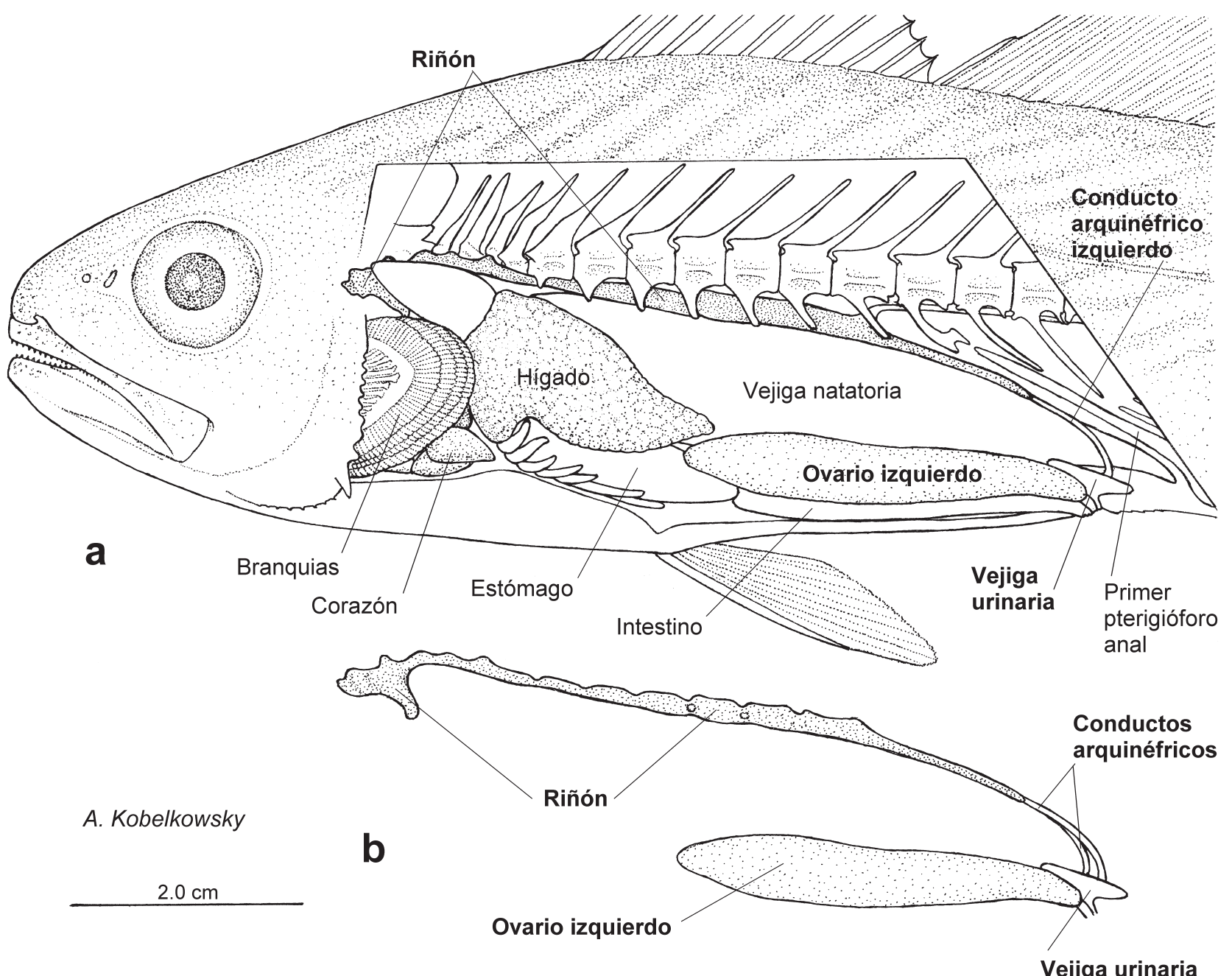

Figura 1. Localización del sistema urogenital de Bairdiella chrysoura. a, organografía de la cavidad visceral en vista lateral izquierda; b, sistema urogenital aislado de la hembra.

en Achirus linetaus (Fig. 4 d), o doble en las especies de Symphurus.

7. Dimorfismo sexual de los conductos y orificios urogenitales. En las hembras de la mayoría de las especies de teleósteos, que se examinaron, el conducto urinario común se abre al exterior mediante el orificio urinario de manera independiente al orificio genital femenino o gonoporo, como se ilustra en Astyanax fasciatus (Fig. 5 a) y Bairdiella chrysoura (Fig. 5 b). Una excepción es Gambusia regani (Fig. 5 c), en la que se forma un seno urogenital al cual se abren tanto el conducto urinario común como el gonoducto femenino.

En los machos de todas las especies de teleósteos examinadas, el conducto urinario común se une al conducto espermático común, formando el conducto urogenital, que generalmente es corto y se abre al exterior mediante el orificio urogenital (Fig. 5 a, b, c).

En varias especies de teleósteos, el orificio urinario de las hembras se encuentra en la punta de una papila urinaria, y el orificio urogenital de los machos en la punta de una papila urogenital. En Gobionellus oceanicus la papila de las hembras es redondeada, y la de los machos triangular (Fig. 5 d). En las hembras de Citharichthys spilopterus la papila urinaria es cónica y lleva surcos finos, mientras que en los machos la papila urogenital lleva un verticilo de pequeños lóbulos (Fig. 5 e). 

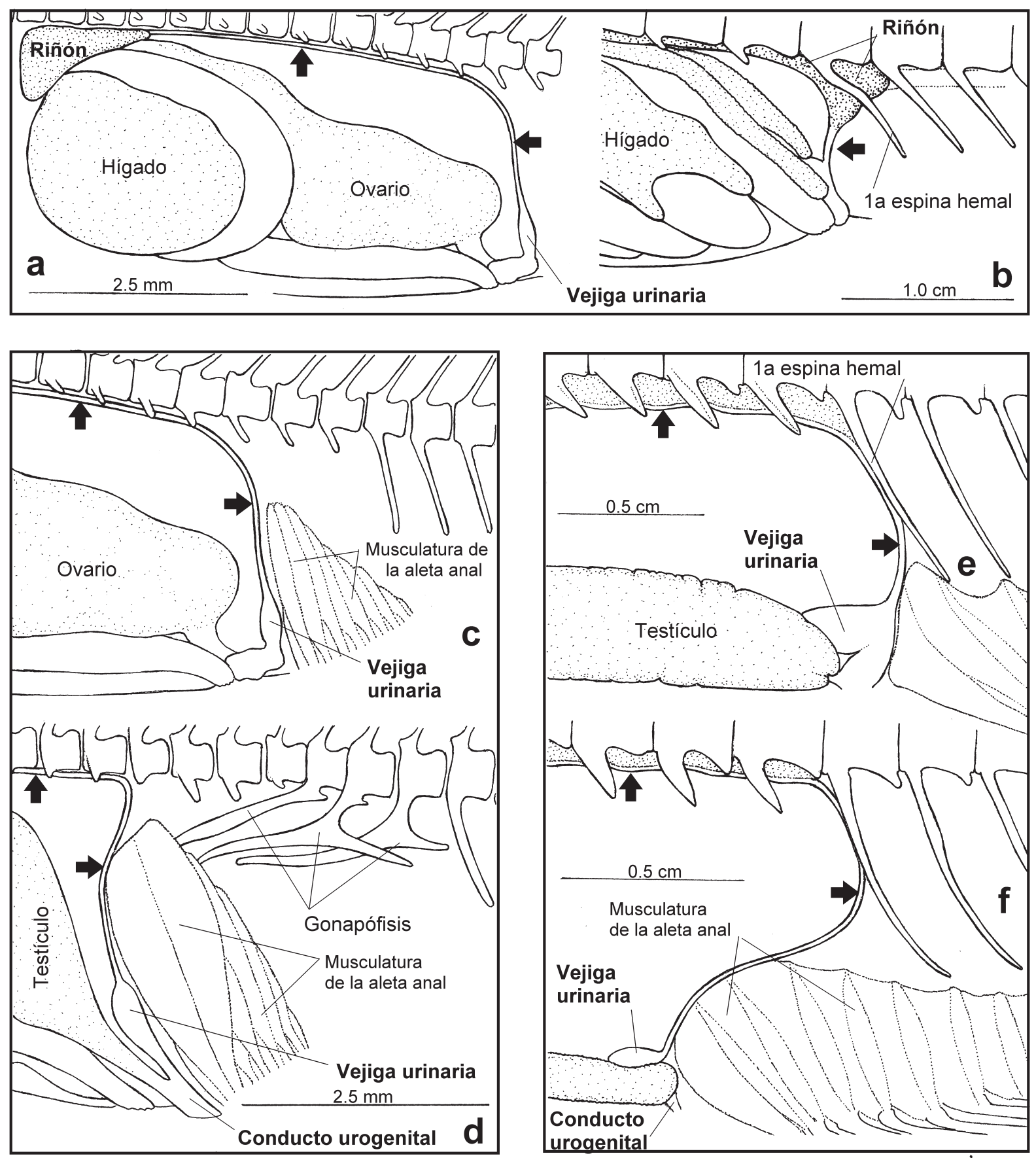

Figura 2. Longitud relativa de los conductos arquinéfricos. Vista lateral izquierda de la cavidad visceral. a, hembra de Gambusia regani; b, macho de Gobionellus oceanicus; c, hembra de Gambusia regani; d, macho de Gambusia regani; e, macho de Membras vagrans, f, macho de Menidia beryllina.

8. Formación de la cloaca. En Diodon hystrix, Chilomycterus schoepfii, Sphoeroides testudineus y Sphoeroides spengleri se reconoce una cloaca a la que se abren el ano y la papila urogenital.

\section{Discusión}

Mientras que en las hembras de los condríctios (peces cartilaginosos) los conductos arquinéfricos se perdieron y 


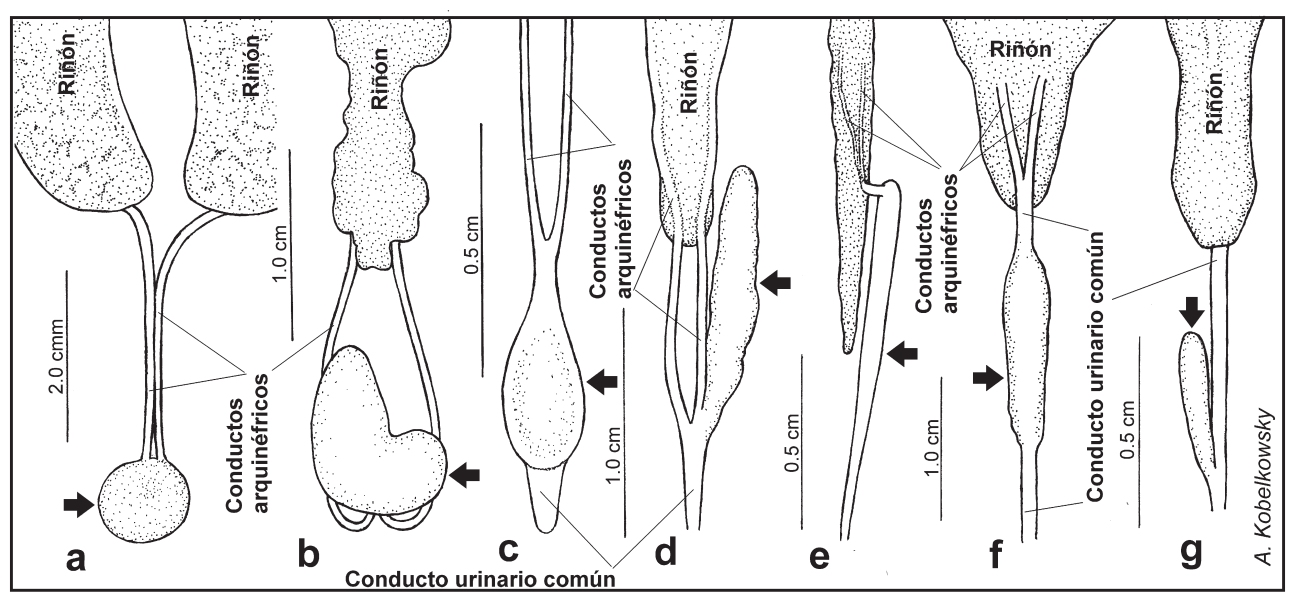

Figura 3. Diversidad de los conductos urinarios y la vejiga urinaria. a, vista ventral de Diodon hystrix; b, vista ventral de Bairdiella ronchus; c, vista dorsal de Goodea atripinnis; d, vista ventral de Opsanus beta; e, vista ventral de Elops saurus; f, vista ventral de Cathorops melanopus; g, vista ventral de Astyanax fasciatus.

en los machos se transformaron en conductos deferentes, en los petromizontiformes (lampreas) y los teleósteos (peces óseos superiores) se han conservado (Romer, 1962). En las lampreas los conductos arquinéfricos forman el borde ventral de los riñones mesonéfricos (Morris, 1972). En ambos sexos de los condríctios la orina es conducida por los conductos urinarios accesorios (Gilbert, 1973).

En el presente trabajo se define como un patrón morfológico del sistema de conductos excretores de los teleósteos el siguiente: las nefronas se conectan a los conductos colectores y éstos a los conductos arquinéfricos, localizados cerca de los bordes del riñón, los cuales se desprenden de su extremo posterior, y después de un tramo se conectan directa o indirectamente a la vejiga urinaria; ambos conductos arquinéfricos se unen entre sí, formando el conducto urinario común, el cual en las hembras se abre al exterior mediante el orificio urinario y en los machos se une al conducto espermático común, para formar el conducto urogenital, que se abre al exterior mediante el orificio urogenital.

Como resultado del análisis anatómico de 70 especies de teleósteos, correspondientes a 62 géneros, 39 familias y 20 órdenes, se reconoce una relativamente alta diversidad de situaciones morfológicas del sistema de conductos excretores de los Teleostei.

Mientras que en el patrón morfológico de los teleósteos los conductos colectores se encuentran dentro del riñón, en la anguila Ophichthus gomessi, varios de estos sobresalen de la superficie, antes de unirse a los conductos arquinéfricos.

Se determina una relación inversa entre la longitud del riñón y la longitud de los conductos arquinéfricos, ejemplificada con Gambusia regani, en que los riñones son cortos y los conductos arquinéfricos son largos y con Gobionellus oceanicus que tiene el riñón largo y los conductos arquinéfricos cortos.

Sin embargo, la longitud de los conductos arquinéfricos puede también estar relacionada con la modificación de la forma de la parte posterior de la cavidad visceral, debida al "adelantamiento" de la aleta anal, sus pterigióforos y su musculatura. Esta situación morfológica se reconoce en la especie examinada de la familia Poeciliidae (Gambusia regani) y en una de Atherinopsidae (Menidia beryllina).

En la mayoría de los teleósteos examinados el diámetro de los conductos arquinéfricos es relativamente reducido; sin embargo, destaca la condición de Synodus foetens (Synodontidae), en la que tanto los conductos arquinéfricos, como el conducto urinario común son tan gruesos como la vejiga urinaria.

La trayectoria de los conductos arquinéfricos en los teleósteos se interpreta como el recorrido más corto desde el riñón hasta la vejiga urinaria, rodeando por alguna parte a la vejiga gaseosa o natatoria. Así, la orientación hacia atrás y hacia abajo de los conductos arquinéfricos hasta alcanzar la vejiga urinaria, se reconoce como un patrón general de los teleósteos. Durante esta trayectoria, dichos conductos hacen contacto con la primera espina hemal y el primer pterigióforo anal y su correspondiente musculatura. Sin embargo, en las especies donde la vejiga gaseosa se prolonga más allá del ano y las aberturas urogenitales, los conductos arquinéfricos rodean este órgano por uno o ambos lados.

Se define como un patrón morfológico general de los teleósteos, la fusión de los 2 conductos arquinéfricos en 


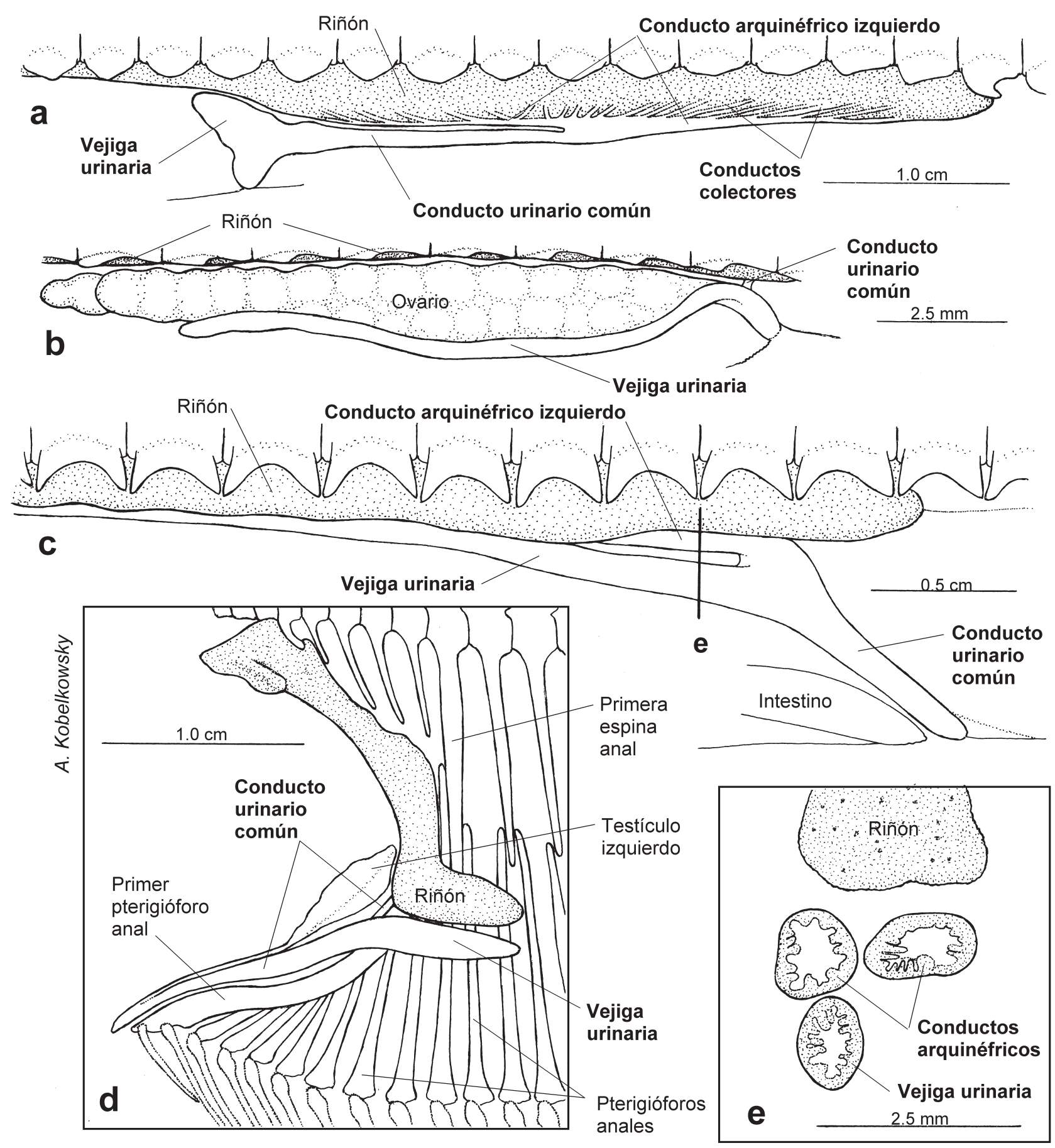

Figura 4. Diversidad de los conductos urinarios y la vejiga urinaria. Vista lateral izquierda. a, Ophichthus gomessi; b, Syngnathus scovelli; c, Synodus foetens (sección transversal correspondiente a la figura e); d, Achirus lineatus; e, corte transversal del sistema excretor de Synodus foetens. 

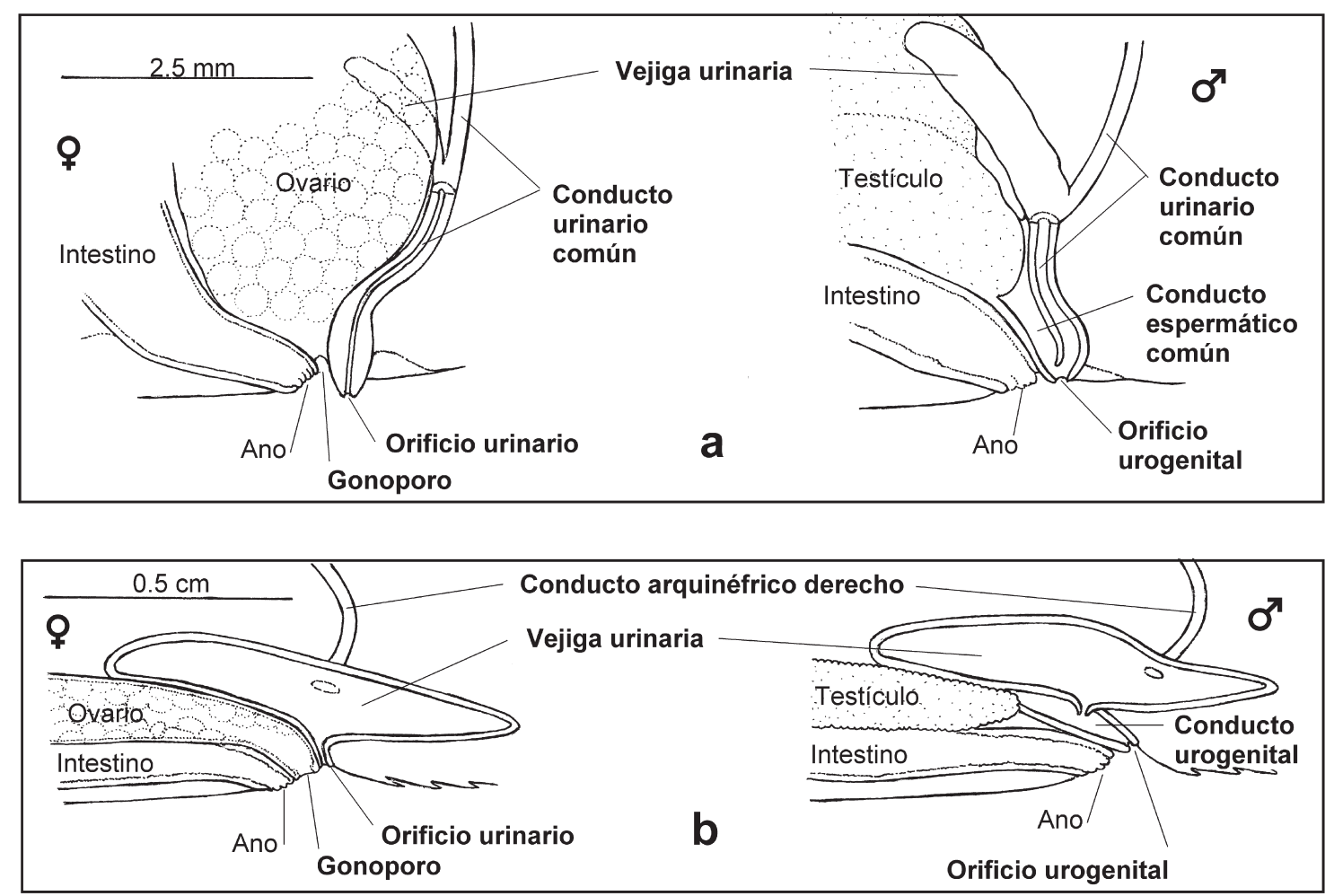

A. Kobelkowsky
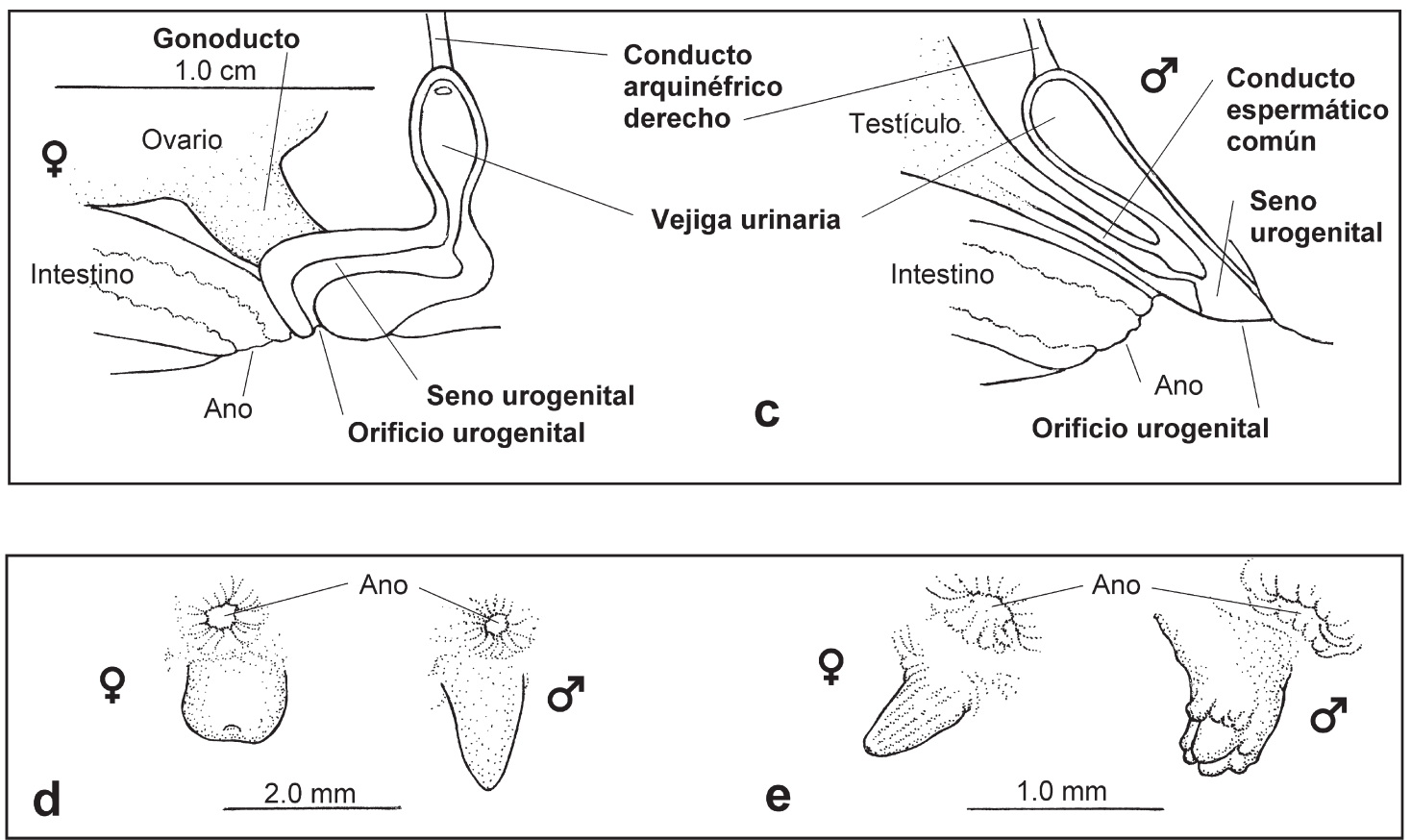

Figura 5. Dimorfismo sexual de los conductos urinarios y urogenitales. Vista lateral izquierda del corte sagital de la porción distal de los conductos. a, hembra y macho de Astyanax fasciatus; b, hembra y macho de Bairdiella chrysoura; c, hembra y macho de Gambusia regani; d, Papilas urogenitales de Gobionellus oceanicus; e, papilas urogenitales de Citharichthys spilopterus. 
el denominado conducto urinario común. En el presente trabajo, se reconoce una diversidad de sitios donde se realiza la fusión.

La condición morfológica más frecuente, consistente en la separación entre sí de los conductos arquinéfricos en el tramo entre el riñón y la vejiga urinaria, o su fusión en el punto de contacto con la misma, se identificó en los Atherinomorpha y los Percomorpha, con excepción de la mayoría de los Pleuronectiformes. En segundo término, se identifica como frecuente la condición en la que ambos conductos arquinéfricos se fusionan dentro del riñón o en su superficie ventral, partiendo del mismo el conducto urinario común, y que corresponde a la mayoría de los Siluriformes (con excepción del representante de los Pimelodidae) y de los Pleuronectiformes (con excepción de los Cynoglossidae).

La forma más frecuente de la vejiga urinaria, consistente en un abultamiento simple o bilobular, la cual se correlaciona con la condición de los conductos arquinéfricos no fusionados entre sí antes de la vejiga.

En el presente trabajo se considera que el "adelantamiento" de la aleta anal en ciertos grupos de teleósteos afecta en cierto grado la morfología de los conductos urinarios.

También se reconoce una relación morfológica entre la posición adelantada de la aleta anal de ciertos teleósteos y la longitud y orientación de los conductos excretores. Mientras que el "adelantamiento" de la aleta anal de los machos de los Poeciliidae solamente reduce la longitud de los conductos arquinéfricos, en los órdenes Anguilliformes y Pleuronectiformes produce una inversión de la orientación del conducto urinario común, ya que los orificios anal y urogenitales quedan en una posición anterior a la que tienen en en la mayoría de los teleósteos.

La orientación hacia adelante del conducto urinario común de Anguilla anguilla, que está ilustrada por Gérard (1954), y que en el presente trabajo se describe en Ophichthus gomessi es probablemente el patrón morfológico de los Anguilliformes.

Se reconoce un patrón morfológico del sistema de conductos urogenitales en el orden Pleuronectiformes, consistente en la "inversión" de la orientación de los mismos, la cual es consecuencia del adelantamiento de la aleta anal y la curvatura del primer pterigióforo anal, como lo ilustra Kobelkowsky (2000) en Achirus lineatus, Trinectes maculatus y Gymnachirus texae; Kobelkowsky (2002) en Citharichthys spilopterus, y Kobelkowsky (2004) en Bothus robinsi.

Mientras que la vejiga urinaria en los anfibios y los reptiles es un derivado de la cloaca, y en los mamíferos del alantoides (Romer, 1962), en el presente trabajo se interpreta el origen de la vejiga urinaria de los teleósteos como una modificación del conducto urinario común. Dicho origen se manifiesta en 2 situaciones: como el ensanchamiento de un tramo del conducto urinario común, según se observa en las familias Ariidae, Ictaluridae, Paralichthyidae y Bothidae y como la evaginación del punto de unión de los 2 conductos arquinéfricos en el urinario común, como se observa en la mayoría de los teleósteos examinados.

La forma, tamaño y orientación de la vejiga urinaria del tipo evaginación es diversa; sobresale por su gran longitud en Syngnathus scovelli (Syngnathidae) y Synodus foetens (Synodontidae) y por su orientación hacia atrás y en posición extravisceral en los lenguados de la familia Achiridae, como describe Kobelkowsky (2000).

El dimorfismo sexual del sistema de conductos urinarios en los teleósteos se manifiesta en la relación de los conductos excretores y los reproductores, y en los orificios urinario y urogenital. Se confirma en las hembras de la mayoría de los grupos de teleósteos la independencia entre los conductos excretores y el gonoducto, y en los machos la fusión del conducto urinario común con el espermático común, formando el conducto urogenital. Sin embargo, como excepción al patrón morfológico de los teleósteos, Peden (1972) y Kobelkowsky (2000) describen la presencia del seno urogenital en las hembras de Gambusia (Poeiliidae). El dimorfismo sexual de los orificios se manifiesta en la diferente forma de la papila urinaria y la papila urogenital de algunas especies.

Es conocida la presencia de la cloaca en los ciclóstomos y en los condríctios; en las lampreas está ubicada al inicio del último tercio del cuerpo y en los tiburones y rayas entre las aletas pélvicas. Mientras que en la gran mayoría de los teleósteos, el ano se abre directamente al exteriorde manera independiente de las aberturas excretora, genital y urogenital, en el presente trabajo se reconoce la cloaca en los Tetraodontiformes Diodon hystrix y Chilomycterus schoepfii (Diodontidae), Sphoeroides testudineus y Sphoeroides spengleri (Tetraodontidae). Sin embargo, en Balistes capriscus (Balistidae), la cloaca no se forma.

\section{Literatura citada}

Castillo, R. M. A., M. A. Pérez H. y A. Kobelkowsky D. 1988. Estudio de la biología de Bairdiella ronchus (Pisces: Sciaenidae) de la Laguna de Tampamachoco, Veracruz, México. Universidad y Ciencia 5:69-84.

Chiasson, R. B. 1980. Laboratory anatomy of the perch. W M. C. Brown, Dubuque. 67 p.

Gérard, P. 1954. Organes uro-génitaux. In Traité de Zoologie. Anatomie, Systematique, Biologie, tome XII., P. P. Grassé (ed.). Masson et Cie , Paris. p.15651583. 
Gilbert, S. G. 1973. Pictorial anatomy of the dogfish. University of Washington Press, Seattle and London. $59 \mathrm{p}$.

Kobelkowsky, A. 1999. El sistema urogenital de los géneros Bairdiella y Stellifer (Pisces: Sciaenidae). Revista de la Sociedad Mexicana de Historia Natural 49:153-162.

Kobelkowsky, A. 2000. Sistema urogenital de la familia Achiridae (Pisces: Pleuronectiformes) del Golfo de México. Hidrobiológica 10: 51-60.

Kobelkowsky, A. 2002. Osteología del lenguado Citharichthys spilopterus (Pisces: Pleuronectiformes). Anales del Instituto de Biología, Universidad Nacional Autónoma de México, Serie Zoología 73:53-65.

Kobelkowsky, A. 2004. Sexual dimorphism of the flounder Bothus robinsi (Pisces: Bothidae). Journal of Morphology 260:165-171.

Kobelkowsky, A. 2005a. General anatomy and sexual dimorphism of Goodea atripinnis (Teleostei: Goodeidae). In Viviparous fishes, M. C. Uribe and H. J. Grier (eds.). New Life, Homestead, Florida. p. 483498.

Kobelkowsky, A. 2005b. El sistema excretor de Astyanax fasciatus (Teleostei: Characidae). Vertebrata Mexicana 17:1-6.

Kobelkowsky, A. y A. Alva-García. 2000. Anatomía sexual de Gambusia regani (Pisces: Poeciliidae). Anales del Instituto de Biología, Universidad Nacional Autónoma de México, Serie Zoología 71: 133-142.

Lagler, K. F., J. E. Bardach, R. R. Miller y D. R. May
Passino. 1977. Ictiología. AGT, México, D.F. 489 p.

Morris, R. 1972. Osmorregulation. In The biology of lampreys, vol. 2. M. H. Hardisty y I. C. Potter (eds.). Academic, London and New York. p. 193-239.

Moyle, P. B. y J. J. Cech. 1988. Fishes. An introduction to ichthyology, Prentice Hall, Englewood Cliffs, New Jersey. 559 p.

Nagahama, Y. 1983. The functional morphology of teleost gonads. In Fish physiology, W. S. Hoar y D. J. Randall (eds.) Academic. p. 223- 275.

Nelson, G. G. 1975. Anatomy of the male urogenital organs of Goodea atripinnis and Characodon lateralis (Atheriniformes: Cyprinodontoidei), and G. atripinnis courtship. Copeia 1975: 475-482.

Nelson, J. S. 1994. Fishes of the world. Wiley, New York. $600 \mathrm{p}$.

Ogawa, M. 1961. Comparative study of the external shape of the teleostean kidney with relation to phylogeny. Scientific Reports. Tokyo Kyoiku Daigaku B10. p. 6168.

Peden, A. E. 1972. Differences in the external genitalia of female gambusine fishes. Southwest Naturalist 17:265-272.

Romer, A. S. 1962. The vertebrate body. Saunders, Philadelphia. 475 p.

Rosen, D. E. y M. Gordon. 1953. Functional anatomy and evolution of male genitalia in poeciliid fishes. Zoologica 38:1-47, IV plates.

Young, J. Z. 1962. The life of vertebrates. Clarendon, Oxford. 820 p. 